THURSDAY, JANUARY 29, 1880

\section{THE FUNDAMENTAL DEFINITIONS AND PROPOSITIONS OF GEOMETRY, WITH ES- PECIAL REFERENCE TO THE SYLLABUS OF THE ASSOCIATION FOR THE IMPROVE- MENT OF GEOMETRICAL TEACHING}

I DESIRE to offer some suggestions respecting the 1 form and arrangement of the elementary definitions and propositions of the Euclidian geometry. It has appeared to me that the recent German textbooks upon the subject have made a great improvement upon the older system, as developed in the works of Euclid and Legendre. I have but recently obtained the "Syllabus of the Association for the Improvement of Geometrical Teaching" and compared it with the corresponding parts of a summary of my own, the latter still in an inchoate state.

I now take the liberty of making some remarks on a few points on which I should be greatly pleased to know the views of those interested. In making them, however, no attempt will be made to go below the fundamental conceptions of the subject which are taken for granted in ordinary textbooks. It may be assumed that there is a general agreement that these conceptions are to be taken for granted, and that the only question is respecting their form and arrangement. One general remark may not, however, be out of place. The aim of elementary geometry is to present its definitions and propositions in a perfectly logical arrangement, so that each definition shall be a complete description, and nothing more, and each proposition be founded strictly on definitions and axioms. It may be doubted whether this perfect ideal is attainable. It might be claimed that our elementary conceptions of relations in space have been derived from experience by processes of abstraction and generalisation, in which no logical order was followed, and that it is impossible to arrange them with that perfect unity which logical method aims at. However this may be, it will, I think, be conceded on all sides that all our systems have hitherto been mere approximations to an ideal which no one has actually reached.

In framing a geometrical definition three different ob. jects may be aimed at.

r. To express our fundamental conceptions of the thing defined in the most accurate form possible.

2. To specify those qualities which most completely differentiate the thing defined from all other things.

3. To describe its axiomatic properties, or those which are subsequently used in demonstrating propositions relating to it.

We thus have three tests which we may apply to a definition and which may lead to different judgments of it. In most cases the same definition will be reached which ever object we have in view. The only concept the definitions of which can be separately classed under all three heads is, so far as I have noticed, that of a straight line. The fundamental quality of a straight line as we conceive of it $i$, I think, that of symmetry, or similarity of properties with respect to space on all sides of it. A line which is throughout its whole length perfectly symmetrical, having no properties on one side which VOL. XXI.-No. 535 it does not equally possess on all other sides, is a straight line. A curve is concave on one side and convex on another. The definition of Simpson's Euclid that a straight line lies evenly between its extreme points, may be considered as an attempt to formulate this conception of symmetry.

The definition which most completely differentiates a straight line from all others is that of some editions of Euclid and Legendre as the shortest distance between two points. It is to be remarked, however, that neitber of these properties is directly made use of in demonstrating the subsequent theorems of geometry. The axiomatic definition of a straight line, if I may be allowed to use the expression, is that of Playfair's Euclid, as being lines which must coincide throughout if they coincide in two points.

Quite similar to that is Definition V. of the Syllabus. This class of definitions, or the axioms in which they are embodied, include the only ones which serve as a basis for the subsequent theorems of geometry.

It is to the definition of plane figures given in the Syllabus that the attention of those interested in this subject is especially asked.

The following are extracts from the Syllabus :-

"Def. VII.-A plane figure is a portion of a plane surface inclosed by a line or lines.

"Def. VIII.-A circle is a plane figure contained by one line, which is called the circumference, \&c.

"Def. XXII.-A plane rectilineal figure is a portion of a plane surface inclosed by straight lines.

"Def. XXVIII.-A triangle is a figure contained by three straight lines."

These definitions agree with those of the old geometry in defining plane figures as inclosed portions of a plane surface. It seems to me that in no part of geometry is greater reform needed than in this.

Figures on a plane surface should, it seems to me, be defined as lines simply, and not as portions of the surface. The following are some of the objections against the old and in favour of the new system of definition :--

I. By Definition VII, as quoted above, an ellipse is a plane figure because it incloses a portion of a plane surface, but a parabola or byperbola is not. Three straight lines may form a figure, but two cannot. But if we form a figure of three straight lines we must cut off all those portions of each line which lie outside of its intersection with the other two as forming no part of the figure.

2. In the modern synthetic geometry figures are considered in a more general way as formed of lines. A triangle, for instance, is a combination of three indefinite straight lines. To this we may, if we please; add the restriction that no two shall be parallel, and that all three shall not pass through a point. The quadrilateral is a combination of four such indefinite lines, to which again if necessary, may be applied the restriction that no then shall be parallel or pass through a point; the circle also becomes the line, not the inclosed space The quadrilateral the student, whose ideas of sucb figures are only those of the elementary geometry, passes to the study of the higher geometry, he is obliged to form a new set of comceptions for the same terms; so great a change, for instance, as substituting the conception of three indefinite straight lines for that of a triangular piece of paper. He reads of 
the intersection of circles, and must understand that it is something radically different from any intersection of the two round planes which he has been taught to consider as circles.

The same change must be carried into space of three dimensions. Studies of what in the elementary geometry have been termed solids, when made by modern mathematicians, are not studies of solids but surfaces. An ellipsoid in modern mathematics is not a solid but a surface. Of course we cannot reject the conception of an inclosed area, but this area must be regarded as something distinct from the figure itself, just as we regard the perimeter as something different. I do not see that anything but good will result from the change here proposed.

In Definition XI. the idea of a "straight" angle is introduced to express the angle of $180^{\circ}$ between two lines emanating from a point in opposite directions. I should like to submit the question whether the term flat angle is any better. The converse of straight is bent or crooked, terms which can hardly be applied to an angle. But the converse of flat is sharp or obtuse, terms which can be so applied. Thus, before seeing the syllabus, the term "flat" appeared to me better than "straight." The introduction of this angle must be regarded as one of the greatest improvements in elementary geometry, but it does not seem to have been introduced into the subsequent theorems of the syllabus in which the old designation of two right angles has been retained without essential alteration. Intimately associated with the fundamental definition of angular measure are the theorems relating to right angles and to the impossibility of straight lines having a common segment; the following three propositions are in fact closely connected.

Two straight lines cannot have a common segment.

All right angles are equal to one another.

If a straight line stands upon another straight line it makes the adjacent angles together equal to two right angles.

The treatment of these propositions by Euclid seems extremely unsatisfactory, and the order in which they are given in the syllabus a great improvement.

Euclid takes the equality of all right angles as an axiom and afterwards proves from it that two straight lines cannot have a common segment. But it seems evident that the equality of right angles depends upon and presupposes the impossibility of a common segment. It must first be self-evident that two straight lines cannot have a common segment before it can be evident that all right angles are equal.

The third of the propositions-just quoted, as considered both by Euclid and Legendre, seem to me unnecessary and circuitous courses of reasoning carried through solely to avoid the conception of the sum of two right angles being itself an angle. This circuit is all the more readily taken from the fact that neither of them has considered it necessary to give a general definition of what shall be meant by the sum of two angles. The syllabus gives this definition and from it alone, without any reasoning whatever, it follows that the sum of the two angles referred to is a flat angle.

As an additional illustration of the simplicity introduced by the consideration of the flat angle we may take
Theorem XXVI. of the syllabus, that the interior angles of any polygon, together with four right angles, are equal to twice as many right angles as the figure has sides. In the new notation we would say that the sum of the interior angles of the polygon is equal to a number of flat angles two less than the polygon has sides, an obvious simplification.

With reference to Definition XII. I would suggest the question whether it would not be better to reserve the term "adjacent angles" for the pair of angles which a straight line makes with another at the point of meeting. We might call these supplementary angles, but the term is suggestive not simply of an arrangement of the two angles but of any pair of angles, wherever or however situated, which together make a flat angle. We certainly need some term to correspond with the Nebenwinkel of the Germans, and I know of none in our geometry.

In Theorem VI. of the syllabus, which is the same as as Proposition V. of Euclid, namely, "The angles at the base of an isosceles triangle are equal to one another," the syllabus suggests a different demonstration from that of Euclid. The extreme complication of the demonstration given by Euclid is very striking, and it will be interesting to see how it arose. Apparently Euclid wished to avoid the conception of turning a figure over and applying it to itself. But the validity of this turning over is presupposed in the demonstration of the theorem, for without it the equality of two triangles having two sides and the included angle equal would be true only for triangles in which the two sides are similarly situated. This question is of especial interest when we apply it to the corresponding case of two equal solid bodies which are mutually obverted or in other words each of which is represented by the image of the other seen in a looking-glass. Are we entitled to assume that two such bodies are identically equal when it is impossible to bring them into coincidence? The only reason why we cannot bring them into coincidence is that our space is confined to three dimensions. Could we open out a fourth dimension in space the one body could, by simple rotation through $180^{\circ}$, be brought into the form of the other and thus made identically equal to it. A man by turning a properly directed somersault in such space would come back into our natural Euclidian space, turning right side left without the mutual arrangements of the parts of his body, even to the minutest atoms, undergoing any change whatever in their relative positions; and therefore without any change, so far as we could see, in the performance of the vital functions. But as a fourth dimension is necessary to the actual performance of such an obversion, so in plane geometry, the third dimension is necessary to the obversion of a plane figure. The syllabus, and so far as I know all the elementary geometries in English are silent on the validity of this process.

The question whether Theorems X. and XI. that the greater side of every triangle is opposite the greater angle, and the greater angle opposite the greater side, should be regarded as independent and demonstrating in entirely different ways is interesting. Since only one side and one angle can be in the relation of opposition how is it possible that the one theorem should be true without the other? Does not one theorem follow from the other by the rule of identity, and]can they not be 
combined into the single theorem that the greater side and the greater angle are opposite each other?

SIMON NEWCOMB

\section{THE SCIENCE OF STATESMANSHIP}

DOLITICAL science and politics are two very different 1 things; some progress has been made in methodising the facts and inductions of political economy, but politics is still little more than a chaos of party prejudices and personal invective. Yet there is surely no reason why political action, the conduct of the State, should not be guided by scientific method quite as much as the conduct of a scientific exploring expedition such as that which has so recently sailed over the North-East Passage. Prof. Nordenskjöld's feat is one of the finest instances of scientific prediction based on ascertained data that we know of, and we would recommend it to Sir William Harcourt's consideration when he contemplates taking part in another political "agitation." Sir William has succeeded in getting such a firm grasp of the real nature of scientific method, and he applied it so wittily and so well in his recent Birmingham address that we would advise him to follow out this line in real earnest. So thoroughly does he seem to understand the method of scientific research and scientific prediction, and so ably, although only in sport and to banter his opponents, did he expound it, that we think science has lost in him a successful worker. To this loss we could resign ourselves if Sir William would set himself to rescue politics from its present degraded position as a mere theatre for party strife, and to elevate it into something like a science of national life and progress. He must have taken considerable pains to obtain his knowledge of the method and uses of the Nautical Almanac; his natural mistake as to its editorship we can overlook. As to the truth of his application of the method of the almanac to the construction of a Conservative Almanac, "after a careful induction from the conduct of Tory government," we have nothing to do here; its ingenuity is amusing. With the following remarks, however, men of all parties cannot but agree :-

"Prediction in politics is not a matter of choice, but of necessity. If public men are not fatalists like the statesmen of our darling Islam, they are bound to foresee and foretell the consequences of their action by which the fortunes of the country are determined. As the predictions prove true or false so will they be judged, for political prophecy, founded upon correct observation and just inference, is nothing else but the science of statesmanship itself."

Here Sir William has struck a vein which might be worked out to the elevation of politics, and with real good to the country. It is, we believe, regarded as an incontrovertible axiom in British politics, that government by party is the surest method of securing the most efficient conduct of public affairs. This point we shall not discuss; but we venture to think that if our political leaders were to give their serious attention to the method indicated above, party differences would be fewer than they are, and party strife less bitter, while the objects supposed to be aimed at by all constitutional governments would be much more effectually and rapidly accomplished.

At present, to judge from the public utterances of our members of parliament and by the results achieved by which ever party may be in power, party government consists mainly in strenuous efforts made by each party either to keep or to obtain place and power; this is accomplished by means of what are called "agitations," the great object of which seems to be to agitate the people into the belief that the agitators are angels from heaven who have the good of the nation disinterestedly at heart, while their opponents are quite the reverse, the only object of the latter being, it is declared, to send the nation to the custody of the person whose emissaries they are.

There are one or two eminent men of science in parliament, but no one of either party ever seems to think of looking at any measure or any line of conduct apart from party bias, and solely as a matter for scientific consideration. It seems enough to damn a measure at once in the eyes of one party, that it originates with their opponents. This is both unscientific and irrational, and can never lead to the best results. The same laws that influence the development of the individual influence the real progress of the nation, and it is only by honest investigation on strictly scientific principles that these laws can be discovered. It is thus that they have been discovered and expounded by Mr. Darwin and his followers in the case of individual organisms, and we would commend to Sir William Harcourt the study of Mr. Darwin's works, if he really desires to arrive at the true principles of scientific statesmanship. One of the great charms of Mr. Darwin's works to the man of science is their perfect candour and fairness. Not only does he adduce all the arguments he can muster in favour of any position or hypotheses he may be considering, but with equal fulness and candour does he treat all, according to his lights, that might be adduced against it, balancing the one series of arguments against another, not in the style of a special pleader, but after the manner of a judge whose sole aim is to discover the truth. Here is a specimen of the method followed by Mr. Darwin, showing his ingenuity in imagining objections to his own theories and thus putting arguments into the mouths of his opponents. We quote from the "Origin of Species" (I860, p. 462):-

"As on the theory of natural selection an interminable number of intermediate forms must have existed, linking together all the species in each group by gradations as fine as our present varieties, it may be asked, Why do we not see these linking forms all around us? Why are not all organic beings blended together in an inextricable chaos? With respect to existing forms, we should remember that we have no right to expect (excepting in rare cases) to discover directly connecting links between them, but only between each and some extinct and supplanted form. Even on a wide area, which has during a long period remained continuous, and of which the climate and other conditions of life change insensibly in going from a district occupied by one species into another district occupied by a closely allied species, we have no just right to expect often to find intermediate varietics in the intermediate zone. For we have reason to believe that only a few species are undergoing change at any one period; and all changes are slowly effected. I have also shown that the intermediate varieties which will at first probably exist in the intermediate zones will be liable ta be supplanted by the allied forms on either hand; and the latter, from existing in greater numbers, will generally be modified and improved at a quicker rate than the intermediate variecies, which exist in lesser numbers; 